PROCEEDINGS OF THE

AMERICAN MATHEMATICAL SOCIETY

Volume 129, Number 5, Pages 1289-1297

S 0002-9939(00)05667-7

Article electronically published on October 24, 2000

\title{
QUASITILTED EXTENSIONS OF ALGEBRAS I
}

\author{
FLÁVIO ULHOA COELHO, MARIA IZABEL R. MARTINS, AND \\ JOSÉ ANTONIO DE LA PEÑA
}

(Communicated by Ken Goodearl)

\begin{abstract}
Let $A$ be a connected finite dimensional $k$-algebra, and let $M$ be a nonzero decomposable $A$-module such that the one-point extension $A[M]$ is quasitilted. We show here that every nonzero indecomposable direct summand of $M$ is directing and $A$ is a tilted algebra.
\end{abstract}

Quasitilted algebras were introduced in 8 as a natural generalization of tilted algebras. Let $k$ be an algebraically closed field, and let $\mathcal{H}$ be a hereditary abelian $k$-category. Then a finite dimensional $k$-algebra $A$ is called a quasitilted algebra if $A=\operatorname{End}_{\mathcal{H}} T$ for a tilting object $T \in \mathcal{H}$. In case $\mathcal{H}=\bmod H$ for $H$ a hereditary algebra, then $A$ is a tilted algebra. Equivalently, $A$ is quasitilted if and only if gldim. $A \leq 2$ and for every indecomposable $A$-module $X$, either the projective dimension $\operatorname{pd}_{A} X \leq 1$ or the injective dimension $\operatorname{id}_{A} X \leq 1$; see [8].

Much work has been done recently on quasitilted algebras. Relevant to our considerations will be the papers $[3,5,7,8,11,13$. The problem of studying conditions for a one-point extension $A[M]$ to be quasitilted was already posed in [8]. It is not difficult to see that, if $A[M]$ is quasitilted, so is the algebra $A$ [8] (III.2.3)]. Interest in the problem is clear since every basic nonsimple quasitilted algebra $B$ can be written as a one-point extension $B=A[M]$ of some smaller quasitilted algebra $A$.

In this work we consider the case in which the module $M$ above decomposes. To state our main result we need to recall that an indecomposable $A$-module $X$ is said to be directing if there is no path of nonzero, nonisomorphism maps $X=X_{0} \rightarrow$ $X_{1} \rightarrow X_{2} \rightarrow \cdots \rightarrow X_{s}=X$ between indecomposable modules.

Theorem. Let $A$ be a connected finite dimensional $k$-algebra, and let $M$ be a nonzero decomposable A-module such that the one-point extension $A[M]$ is quasitilted. Then every nonzero indecomposable direct summand of $M$ is directing. Moreover, $A$ is a tilted algebra.

One of the main features used in the proof of this theorem is the remark that for an algebra $B=A[M]$ with $M$ a nonzero decomposable module, the first Hochschild cohomology $H^{1}(B)$ does not vanish. In particular, there are finite Galois coverings $\tilde{B} \rightarrow B$ defined by the action of a finite abelian group $G$, such that $\tilde{B}$ is given as a multiple one-point extension of an algebra of the form $A \times A \times \cdots \times A$. Moreover, if

Received by the editors October 9, 1998 and, in revised form, August 11, 1999

2000 Mathematics Subject Classification. Primary 16G70, 16G20, 16 E10.

Key words and phrases. Quasitilted algebras, one-point extensions, directing modules.

(C)2000 American Mathematical Society 
the order of $G$ is a unit in $k$, then $B$ is quasitilted if and only if $\tilde{B}$ is; see [8, 12, 14. Applications of this technique yield information on the possible modules $M$ for which the extension $A[M]$ is quasitilted.

We briefly summarize the contents of the paper. In section 1 we recall some basic facts on quasitilted algebras and prove some useful lemmas. In section 2 we consider Galois coverings of algebras $A[M]$ with $M$ a decomposable $A$-module. Section 3 is devoted to the proof of the main Theorem.

In a forthcoming publication [4] we shall consider the problem of characterizing the pairs $(A, M)$ of quasitilted algebras $A$ and decomposable $A$-modules $M$ such that $A[M]$ is quasitilted. This work was completed during exchange visits in México-São Paulo. The authors wish to thank their institutions, FAPESP in Brazil and CONACyT in México, for support.

\section{Some Generalities on QUASITILTED ALGEBRAS}

1.1. Along this work, $k$ will denote an algebraically closed field. By an algebra we mean an associative finite dimensional $k$-algebra. Unless otherwise stated, we shall assume that an algebra is basic (that is, $A / \operatorname{rad} A$ is a sum of copies of $k$ ) and connected (that is, $A$ is not of the form $A_{1} \times A_{2}$ for two algebras $A_{1}$ and $A_{2}$ ). For a given algebra $A$, denote by $\bmod A$ the category of finitely generated left $A$-modules, by $\Gamma_{A}$ the Auslander-Reiten quiver of $A$, and by $\tau_{A}$ the Auslander-Reiten translate DTr. We refer the reader to [1] for unexplained notions in representation theory of Artin algebras.

1.2. Let $A$ be a finite dimensional $k$-algebra, and let $X$ be an indecomposable $A$-module. An indecomposable module $Y$ is said to be a predecessor of $X$ if there is a path $Y=Y_{0} \rightarrow Y_{1} \rightarrow \cdots \rightarrow Y_{s}=X$ of nonzero maps between indecomposable modules $Y_{1}, \ldots, Y_{s}$; in this case, we also say that $X$ is a successor of $Y$.

In [8] the full subcategories $\mathcal{L}_{A}$, and let $\mathcal{R}_{A}$ of $\bmod A$ were introduced as follows. The category $\mathcal{L}_{A}$ is formed by those indecomposable modules $X$ such that every predecessor $Y$ of $X$ has $\operatorname{pd}_{A} Y \leq 1$. Dually, $\mathcal{R}_{A}$ is formed by those $X$ such that every successor $Y$ has $\operatorname{id}_{A} Y \leq 1$. For later reference, we recall the following result proven in [5].

Theorem ([5]). Let $A$ be a quasitilted algebra, and let $\Gamma$ be a component of $\Gamma_{A}$ with infinitely many $\tau_{A}$-orbits or containing an oriented cycle.

(i) If $\Gamma$ contains a projective module, then $\Gamma$ is contained in $\mathcal{L}_{A} \backslash \mathcal{R}_{A}$.

(ii) If $\Gamma$ contains an injective module, then $\Gamma$ is contained in $\mathcal{R}_{A} \backslash \mathcal{L}_{A}$.

1.3. Lemma. Let $A$ be a quasitilted algebra, and let $X, Y$ be indecomposable $A$ modules with either $X \in \mathcal{R}_{A}$ or $Y \in \mathcal{L}_{A}$. Assume that $X$ is a predecessor of $Y$. Then we have the following assertions:

(a) [3, 8]: There is an indecomposable module $Z$ and nonzero maps $X \rightarrow Z$ and $Z \rightarrow Y$.

(b) Assume that $X \in \mathcal{R}_{A}$ and that there is a nonsectional path from $X$ to $Y$. Then $Y$ is nonprojective and there exist an indecomposable module $Z$ and nonzero maps $X \rightarrow Z$ and $Z \rightarrow \tau_{A} Y$.

Proof. (b) Assume $X \in \mathcal{R}_{A}$. By (a), it is enough to show that there is a path of nonzero maps from $X$ to $\tau Y$. By hypothesis, there is a nonsectional path of 
nonzero maps of the form

$$
X \rightarrow \cdots \rightarrow N \rightarrow * \rightarrow \tau_{A}^{-} N \rightarrow \cdots \rightarrow Y .
$$

Using (a), we infer that there exists an indecomposable module $U$ and nonzero maps $f: \tau_{A}^{-} N \longrightarrow U$ and $g: U \longrightarrow Y$. Suppose first that $g f \neq 0$. Since $X \in \mathcal{R}_{A}$, $\operatorname{id}_{A} N \leq 1$ and so $Y$ is nonprojective (see [1, (IV.1.16)]). Therefore, there exists a path of nonzero maps from $N$ to $\tau_{A} Y$ and the result is proven in this case. Suppose now $g f=0$. By [6], there exists an indecomposable module $W$ such that $\operatorname{Hom}_{A}\left(\tau_{A}^{-} N, \tau_{A} W\right) \neq 0$ and $\left.\operatorname{Hom}_{A}(W, Y)\right) \neq 0$. Again, since $\operatorname{id}_{A} \tau_{A} W \leq 1$, we get that $Y$ is nonprojective and so there exists a path from $\tau_{A}^{-} N$ to $\tau_{A} Y$. This proves the claim.

1.4. In the introduction, we recalled the notion of indecomposable directing modules. We now recall the definition of an arbitrary directing module. It is not difficult to see that both definitions coincide for indecomposable modules (see 6]). We say that a module $X \in \bmod A$ is directing if there are no paths of nonzero maps between indecomposable modules as follows:

$$
X^{\prime} \rightarrow \cdots \rightarrow \tau_{A} Y \rightarrow * \rightarrow Y \rightarrow \cdots \rightarrow X^{\prime \prime}
$$

where $X^{\prime}$ and $X^{\prime \prime}$ are indecomposable direct summands of $X$.

Lemma. Let $A$ be a quasitilted algebra, and let $X$ be a nondirecting module in $\operatorname{add}\left(\mathcal{L}_{A} \cap \mathcal{R}_{A}\right)$. Then there exists an indecomposable module $Z$ such that $\operatorname{Hom}_{A}(X, Z)$ $\neq 0 \neq \operatorname{Ext}_{A}^{1}(X, Z)$.

Proof. Since $X$ is nondirecting, and using (1.3.b) we infer that there exist indecomposable direct summands $X^{\prime}$ and $X^{\prime \prime}$ of $X$ and an indecomposable module $Z$ such that $\operatorname{Hom}_{A}\left(X^{\prime}, Z\right) \neq 0 \neq \operatorname{Hom}_{A}\left(Z, \tau_{A} X^{\prime \prime}\right)$. Since $\operatorname{pd}_{A} X^{\prime \prime} \leq 1$, we get that $\operatorname{Ext}_{A}^{1}\left(X^{\prime \prime}, Z\right) \cong D \operatorname{Hom}_{A}\left(Z, \tau_{A} X^{\prime \prime}\right) \neq 0$ (see [1]).

1.5. We recall that for an $A$-module $M$, the one-point extension algebra $A[M]$ is by definition the finite dimensional $k$-algebra

$$
A[M]=\left(\begin{array}{ll}
k & 0 \\
M & A
\end{array}\right)
$$

with the usual matrix operations. The category of $A[M]$-modules is equivalent to the category of triples $\left(k^{t}, X, f\right)$, where $X \in \bmod A$ and $f: M \otimes k^{t} \rightarrow X$ is a morphism of $A$-modules; see [15].

A nonsimple quasitilted algebra $B$ is always of the form $A[M]$ for a quasitilted algebra $A$ and an $A$-module $M \in \operatorname{add} \mathcal{L}_{A}$ [8, III.2.3,2.4]. For a $B$-module $Y=$ $\left(k^{t}, X, f\right)$ the following is easy to establish:

(i) [8, III.2.1]: If $\operatorname{pd}_{B} Y \leq 1$, then $\operatorname{ker} f$ is projective and $\operatorname{pd}_{A} X \leq 1$. Moreover, if $\operatorname{pd}_{A}$ Coker $f \leq 1$, then $\operatorname{pd}_{B} Y \leq 1$ if and only if ker $f$ is projective.

(ii) [8 III.2.2]: $\operatorname{id}_{B} Y \leq 1$ if and only if $\operatorname{id}_{A} X \leq 1$ and $\operatorname{Ext}_{A}^{1}(M, X)=0$.

1.6. Part of the statement of the following result is a generalization of [3, (2.1)]. Recall that a projective module is called hereditary if each of its submodules is projective. Let $P$ be a hereditary projective module. Clearly, any predecessor of $P$ is also projective, and so $P$ is directing.

Proposition. Let $A$ be an algebra, and let $M=M_{1} \oplus M_{2}$ be an $A$-module such that $B=A[M]$ is a quasitilted algebra. Assume that $M_{1}$ is not hereditary projective. Then $M_{2}$ is a directing module in add $\left(\mathcal{R}_{A}\right)$. 
Proof. Suppose $M_{2}$ is not a directing module. Then, by (1.4), there exists an indecomposable module $Z$ with $\operatorname{Hom}_{A}\left(M_{2}, Z\right) \neq 0 \neq \operatorname{Ext}_{A}^{1}\left(M_{2}, Z\right)$. Let $0 \neq \beta \in$ $\operatorname{Hom}_{A}\left(M_{2}, Z\right)$.

Since $M_{1}$ is not hereditary projective, then $M_{1}$ has a nonprojective submodule $K$ and consider the short exact sequence $0 \longrightarrow K \longrightarrow M_{1} \stackrel{\alpha}{\longrightarrow} C \longrightarrow 0$. Observe that the module

$$
Y=\left(k, C \oplus Z,\left(\begin{array}{cc}
\alpha & 0 \\
0 & \beta
\end{array}\right): M_{1} \oplus M_{2} \rightarrow C \oplus Z\right)
$$

is indecomposable (compare [3. (1.4)]) and $\operatorname{pd}_{B} Y=2=\mathrm{id}_{B} Y$, a contradiction to the assumption that $B$ is quasitilted. Hence $M_{2}$ is directing.

It remains to show in this case that $M_{2} \in \operatorname{add}\left(\mathcal{R}_{A}\right)$. Suppose this is not the case and let $M_{2}^{\prime}=Y_{0} \stackrel{\beta_{1}}{\longrightarrow} Y_{1} \longrightarrow \cdots \stackrel{\beta_{s}}{\longrightarrow} Y_{s}$ be a chain of nonzero maps between indecomposable modules, where $M_{2}^{\prime}$ is a direct summand of $M_{2}$ and $\operatorname{id}_{A} Y_{s}=2$. Clearly, the indecomposable $B$-module

$$
W=\left(k, C \oplus M_{2},\left(\begin{array}{cc}
\alpha & 0 \\
0 & 1
\end{array}\right): M_{1} \oplus M_{2} \rightarrow C \oplus M_{2}\right)
$$

satisfies $\operatorname{pd}_{B} W=2$ and hence $W \notin \mathcal{L}_{B}$. Moreover, there is a chain of nonzero maps

$$
W \rightarrow\left(k, Y_{0},(0, \pi)\right) \rightarrow\left(k^{\delta_{1}}, Y_{1},\left(0, \beta_{1} \pi\right)\right) \rightarrow \cdots \rightarrow\left(k^{\delta_{s}}, Y_{s},\left(0, \beta_{s} \ldots \beta_{1} \pi\right)\right)
$$

in $\bmod B$, where $\pi: M_{2} \rightarrow M_{2}^{\prime}=Y_{0}$ is the natural projection and $\delta_{i}=1$ if $\beta_{i} \ldots \beta_{1} \pi \neq 0$ and $\delta_{i}=0$ otherwise. Since $\operatorname{id}_{B}\left(k^{\delta_{s}}, Y_{s},\left(0, \beta_{s} \ldots \beta_{1} \pi\right)=2\right.$, then $\hat{Y} \notin \mathcal{R}_{B}$, which is a contradiction.

1.7. Lemma. If $B=A\left[M_{1} \oplus M_{2}\right]$ is a quasitilted algebra, then either

$$
\operatorname{Hom}_{A}\left(M_{1}, \mathcal{L}_{A} \backslash \mathcal{R}_{A}\right)=0 \quad \text { or } \operatorname{Hom}_{A}\left(M_{2}, \mathcal{R}_{A} \backslash \mathcal{L}_{A}\right)=0
$$

Proof. Suppose there exist nonzero morphisms $\alpha: M_{1} \longrightarrow Z_{1}$ with $Z_{1} \in \mathcal{L}_{A} \backslash \mathcal{R}_{A}$, and $\beta: M_{2} \longrightarrow Z_{2}$ with $Z_{2} \in \mathcal{R}_{A} \backslash \mathcal{L}_{A}$. By definition, there are paths of nonzero maps between indecomposable modules

$$
Z_{1}=U_{0} \stackrel{\gamma_{1}}{\longrightarrow} U_{1} \stackrel{\gamma_{2}}{\longrightarrow} U_{2} \longrightarrow \cdots \stackrel{\gamma_{n}}{\longrightarrow} U_{n} \quad \text { with } \operatorname{id}_{A} U_{n}=2
$$

and $V_{m} \longrightarrow \cdots \longrightarrow V_{0}=Z_{2}$ with $\operatorname{pd}_{A} V_{m}=2$. By [3, (1.4)], the $B$-module $Z=\left(k, Z_{1} \oplus Z_{2},\left(\begin{array}{cc}\alpha & 0 \\ 0 & 1\end{array}\right)\right)$ is indecomposable. Observe that there is a path of nonzero maps in $\bmod B$

$$
\left(0, V_{m}, 0\right) \longrightarrow\left(0, V_{m-1}, 0\right) \longrightarrow \cdots \longrightarrow\left(0, V_{0}, 0\right) \longrightarrow Z
$$

with $\operatorname{pd}_{B}\left(0, V_{m}, 0\right)=2$, and therefore $Z \in \mathcal{L}_{B}$. Moreover, there is a path of nonzero maps between indecomposable $B$-modules

$$
Z \longrightarrow\left(k, U_{0},(\alpha, 0)\right) \longrightarrow \cdots \longrightarrow\left(k^{\delta_{n}}, U_{n},\left(\gamma_{n} \cdots \gamma_{1} \alpha, 0\right)\right)
$$

where $\delta_{i}=1$ if $\gamma_{i} \cdots \gamma_{1} \alpha \neq 0$, and $\delta_{i}=0$ otherwise. Therefore $Z \in \mathcal{R}_{\mathcal{B}}$ because $\operatorname{id}_{B}\left(k^{\delta_{n}}, U_{n},\left(\gamma_{n} \cdots \gamma_{1} \alpha, 0\right)\right)=2$, a contradiction to the fact that $B$ is a quasitilted algebra. 
1.8. The following result from [13] will be essential in our considerations.

Theorem ([13]). Let $A$ be a quasitilted algebra accepting an indecomposable directing module lying in $\mathcal{L}_{A} \cap \mathcal{R}_{A}$. Then $A$ is a tilted algebra.

Sketch of proof. Consider the full subcategory $\mathcal{H}$ of the derived category $D^{b}(\bmod A)$ generated by $\mathcal{R}_{A}$ and the shift $\left(\mathcal{L}_{A} \backslash \mathcal{R}_{A}\right)[1]$ of $\mathcal{L}_{A} \backslash \mathcal{R}_{A}$ as in [8]. Then $\mathcal{H}$ is a hereditary category with a tilting object. Moreover, a directing indecomposable module $X \in \mathcal{L}_{A} \cap \mathcal{R}_{A} \subset \bmod A$ remains directing as an object in $\mathcal{H}$. A recent result in [7] implies that $\mathcal{H}$ is derived equivalent to $\bmod H$ for $H$ a hereditary algebra. It follows easily that $A$ is a hereditary algebra.

1.9. We are ready to show part of the result stated in the introduction.

Theorem. Let $A$ be an algebra, and let $M$ be a nonzero decomposable A-module such that $A[M]$ is a quasitilted algebra. Then $A$ is tilted.

Proof. Let $M=\bigoplus_{i=1}^{m} M_{i}$ be a decomposition into indecomposable modules. Since $M_{1} \in \mathcal{L}_{A}$, then, by (1.8), we may assume that $M_{1}$ is not directing or lies in $\mathcal{L}_{A} \backslash \mathcal{R}_{A}$. Then (1.6) implies that $\bigoplus_{i=2}^{m} M_{i}$ is hereditary projective. In particular, $M_{2}$ is a directing module. Again by (1.8), we may assume that $M_{2} \in \mathcal{L}_{A} \backslash \mathcal{R}_{A}$. Let $I$ be an indecomposable direct summand of the injective envelope of $M_{2}$. Since $\operatorname{Hom}_{A}\left(M_{2}, \mathcal{L}_{A} \backslash \mathcal{R}_{A}\right) \neq 0$, by (1.7), we get that $\operatorname{Hom}_{A}\left(M_{1}, \mathcal{R}_{A} \backslash \mathcal{L}_{A}\right)=0$ and therefore $I \in \mathcal{L}_{A}$. The result now follows from [8, (II.3.4)].

\section{Galois coverings of One-Point extension algebras}

2.1. Let $A$ be an algebra and $M_{1}, M_{2} \in \bmod A$. Let $n \in \mathbf{N}$ and consider the cyclic group $\mathbf{Z} /(n)$. In the algebra $A^{(n)}=A \times A \times \cdots \times A(n$ copies of $A)$ consider the modules

$$
X_{i}=\left(0, \ldots, 0, M_{i}, 0, \ldots, 0\right) \quad \text { and } \quad Y_{i}=\left(0, \ldots, 0, \underset{i}{M_{2}}, 0, \ldots, 0\right) .
$$

Call $\hat{B}_{n}$ the iterated one-point extension

$$
\hat{B}_{n}=A^{(n)}\left[X_{1} \oplus Y_{2}\right]\left[X_{2} \oplus Y_{3}\right] \ldots\left[X_{n-1} \oplus Y_{n}\right]\left[X_{n} \oplus Y_{1}\right] .
$$

To fix ideas we explicitly write $\hat{B}_{2}$ :

$$
\hat{B}_{2}=\left(\begin{array}{llll}
k & 0 & 0 & 0 \\
0 & k & 0 & 0 \\
M_{2} & M_{1} & A & 0 \\
M_{1} & M_{2} & 0 & A
\end{array}\right)
$$

which accepts the action of $\mathbf{Z} /(2)$ in a natural way.

We refer the reader to [2, 12, 14] for the general theory of Galois coverings of algebras and categories.

Lemma. There is a Galois covering map $\pi_{n}: \hat{B}_{n} \rightarrow B=A\left[M_{1} \oplus M_{2}\right]$ defined by the action of $\boldsymbol{Z} /(n)$ as a group of automorphisms of $\hat{B}_{n}$.

Proof. We do not write explicitly the (rather obvious) definition of $\pi_{n}$ and the automorphisms of $\hat{B}_{n}$ which yield a group $G=\left\{g_{0}=1, g_{1}, \ldots, g_{n-1}\right\}$ isomorphic to $\mathbf{Z} /(n)$ with the property that $\pi_{n} g_{i}=\pi_{n}$, for $0 \leq i \leq n-1$. Let 
$A=\bigoplus_{i=1}^{m} P_{i}$ be the decomposition into indecomposable $A$-modules, and let $Q=$ $\left(k, M_{1} \oplus M_{2}\right.$, id: $\left.M_{1} \oplus M_{2} \rightarrow M_{1} \oplus M_{2}\right)$ be the indecomposable projective $B$ module which is not an $A$-module. Correspondingly, we get indecomposable projective $A^{(n)}$-modules $P_{j i}=\left(0, \ldots, 0, P_{j}, 0, \ldots, 0\right), 1 \leq j \leq m, 1 \leq i \leq n$, and $Q_{i}=$ $\left(k, X_{i} \oplus Y_{i+1}, \mathrm{id}\right) \in \bmod A^{(n)}\left[X_{i} \oplus Y_{i+1}\right] \subset \bmod \hat{B}_{n}$. The modules $P_{j i}$ and $Q_{i}$ yield a complete set of representatives of the isoclasses of indecomposable projective $\hat{B}_{n^{-}}$ modules. Observe that $G$ may be defined in such a way that $P_{j i}^{g_{t}}=P_{j(i+t)}$ and $Q_{i}^{g_{t}}=Q_{i+t}, 1 \leq j \leq m, 1 \leq i \leq n, 0 \leq t \leq n-1$. It is easy to check that

$$
\begin{aligned}
\bigoplus_{g \in G} \operatorname{Hom}_{\hat{B}_{n}}\left(P_{j i}^{g}, P_{s t}\right) & =\operatorname{Hom}_{A}\left(P_{j}, P_{s}\right), \\
\bigoplus_{g \in G} \operatorname{Hom}_{\hat{B}_{n}}\left(Q_{i}^{g}, Q_{s}\right) & =\operatorname{Hom}_{A}(Q, Q), \\
\bigoplus_{g \in G} \operatorname{Hom}_{\hat{B}_{n}}\left(P_{j i}^{g}, Q_{s}\right) & =\bigoplus_{i=s}^{s+1} \operatorname{Hom}_{\hat{B}_{n}}\left(P_{j i}, Q_{s}\right)=\operatorname{Hom}_{A}\left(P_{j}, M_{1} \oplus M_{2}\right) \\
& =\operatorname{Hom}_{A}\left(P_{j}, Q\right), \\
\bigoplus_{g \in G} \operatorname{Hom}_{\hat{B}_{n}}\left(Q_{i}^{g}, P_{s}\right) & =0=\operatorname{Hom}_{A}\left(Q, P_{s}\right)
\end{aligned}
$$

which shows that $\pi_{n}: \hat{B}_{n} \rightarrow B$ is a covering in the sense of [2].

2.2. Let $B=A\left[M_{1} \oplus M_{2}\right], \hat{B}_{n}$ and $G=\mathbf{Z} /(n)$ be as in (2.1). We shall consider the skew group algebra $\hat{B}_{n}[G]$ whose elements are of the form $\sum_{g \in G} b_{g} g$ with multiplication induced by $(b g)\left(b^{\prime} h\right)=b g\left(b^{\prime}\right) g h$; see [14].

Proposition. Assume that the order $n$ of $G$ is a unit in $k$. Then $\hat{B}_{n}[G]$ is Morita equivalent to $B$. Moreover, $\hat{B}_{n}$ is quasitilted (respectively tilted) if and only if $B$ is.

Proof. In [12, 14] it is shown that the character group $X(G) \cong G$ acts on $B$ and $\hat{B}_{n} \cong B[X(G)]$. Then [14] shows that $\hat{B}_{n}[G]$ is Morita equivalent to $B$. The last statement is shown in [8, III (1.6)] and [14].

Corollary. Let $M=M_{1} \oplus M_{2}$ be a nonzero decomposable module such that $A[M]$ is a quasitilted algebra. Then the algebra

$$
B^{\prime}=\left(\begin{array}{lll}
k & 0 & 0 \\
M_{1} & A & 0 \\
M_{2} & 0 & A
\end{array}\right)
$$

is tilted.

Proof. The algebra $B^{\prime}$ is a convex subcategory of the algebras $C_{n}$ such that $\hat{B}_{n}=$ $C_{n}\left[Z_{1} \oplus Z_{2}\right]$, where $Z_{1} \oplus Z_{2}$ is the corresponding decomposition of $\operatorname{rad} Q_{1}$ defined as in (2.1), where $n \geq 2$. Choosing $n$ adequately, the proposition above implies that $\hat{B}_{n}$ is quasitilted and (1.9) implies that $C_{n}$ is tilted. Therefore $B^{\prime}$ is tilted.

\section{Proof of the theorem}

3.1. We start with some simple remarks concerning the structure of the module category of tilted algebras. 
Lemma. Let $A$ be a tilted algebra with connecting component $\mathcal{C}$ which is neither preprojective nor preinjective. Then $\mathcal{L}_{A} \cap \mathcal{R}_{A} \subset \mathcal{C}$.

Proof. If $\mathcal{C}$ is a regular component, then [8, II (3.1)] implies that $\mathcal{L}_{A} \cap \mathcal{R}_{A}$ is formed by the modules in $\mathcal{C}$. So, suppose $\mathcal{C}$ is not regular.

Claim. If $\mathcal{C}$ contains an injective module, then any $X \in \mathcal{L}_{A}$ has successors in $\mathcal{C}$.

Observe that, in this case, $\mathcal{C}$ contains modules of projective dimension 2. Now, if $X$ is an indecomposable module not in $\mathcal{C}$ but such that $\operatorname{Hom}_{A}(\mathcal{C}, X) \neq 0$, then using the lifting property of the almost split maps, we infer that $X$ is a successor of a module of projective dimension 2, and so $X \notin \mathcal{L}_{A}$. Hence, if $X \in \mathcal{L}_{A}$, then $\operatorname{Hom}_{A}(\mathcal{C}, X)=0$ and $X$ is a predecessor of a module in $\mathcal{C}$. This proves the claim. Dually, if $\mathcal{C}$ contains a projective, then any $X \in \mathcal{R}_{A}$ has predecessors in $\mathcal{C}$. Consequently, if $\mathcal{C}$ contains both projective and injective modules, we infer that $\mathcal{L}_{A} \cap \mathcal{R}_{A} \subset \mathcal{C}$. Suppose now that $\mathcal{C}$ contains injective modules but not projectives. Observe that $A$ is a nonconcealed tilted algebra from a hereditary algebra of wild type. Hence, there exists an indecomposable projective module $P$ not lying in a preprojective component of $\Gamma_{A}$. Let $Y \in \mathcal{L}_{A} \backslash \mathcal{C}$. Using [10, (3.2)] and the fact that $A$ is connected, we infer that $\tau_{A}^{-} Y$ is a predecessor of $P$ and so $Y \notin \mathcal{R}_{A}$. This proves the lemma.

3.2. Lemma. Let $A$ be a tilted algebra, and let $M_{1}$ be a nondirecting indecomposable module.

(a) If $M_{1} \in \mathcal{L}_{A} \backslash \mathcal{R}_{A}$, then the connecting component $\mathcal{C}$ of $\Gamma_{A}$ is not preprojective and any indecomposable projective in $\mathcal{L}_{A} \cap \mathcal{R}_{A}$ belongs to $\mathcal{C}$.

(b) If $M_{1} \in \mathcal{L}_{A} \cap \mathcal{R}_{A}$, then $\mathcal{C}$ is either preprojective or preinjective.

Proof. (a) Since $M_{1} \notin \mathcal{R}_{A}$, there exists an indecomposable projective module $P^{\prime}$ which is a successor of $M_{1}$. Since $M_{1}$ is not directing, $P^{\prime}$ does not belong to a preprojective component in $\Gamma_{A}$. In particular, $\mathcal{C}$ is not preprojective. Now let $P$ be an indecomposable projective in $\mathcal{L}_{A} \cap \mathcal{R}_{A}$. By [5, (6.5)], we have that $P \in \mathcal{C}$ as required.

(b) This follows from (3.1).

3.3. Proposition. Let $A$ be a tilted algebra, and let $M_{1}$ be a nondirecting indecomposable module in $\mathcal{L}_{A} \backslash \mathcal{R}_{A}$. Assume that $A\left[M_{1} \oplus M_{2}\right]$ is a quasitilted algebra. Then $M_{2}=0$.

Proof. Suppose $M_{2} \neq 0$, and let $Y$ be an indecomposable direct summand of $M_{2}$. By (1.6), $Y$ is a hereditary projective module in $\mathcal{L}_{A} \cap \mathcal{R}_{A}$. Consider $\mathcal{C}$ the connecting component of $\Gamma_{A}$. By $(3.2), \mathcal{C}$ is not preprojective and $Y \in \mathcal{C}$. By $(2.2)$, the algebra

$$
B^{\prime}=\left(\begin{array}{lll}
k & 0 & 0 \\
M_{1} & A & 0 \\
M_{2} & 0 & A
\end{array}\right)
$$

is tilted. Consider the indecomposable projective $B^{\prime}$-module $P$ with $\operatorname{rad} P=$ $\left(M_{1}, 0\right) \oplus\left(0, M_{2}\right)$ and indecomposable direct summands $X_{1}=\left(M_{1}, 0\right)$ and $Y^{\prime}=$ $(0, Y)$. The module $X_{1}$ is not directing in $\bmod A^{(2)}$ and $Y^{\prime}$ belongs to a directing component $\mathcal{C}^{\prime}$ of $\Gamma_{A^{(2)}}$ containing infinitely many modules $Z \in \mathcal{C}^{\prime}$ which are predecessors of modules $Y_{i}$ on a sectional path of irreducible maps $Y^{\prime}=Y_{0} \rightarrow Y_{1} \rightarrow$ $\cdots \rightarrow Y_{s}$. Let $\mathcal{D}$ be the component of $\Gamma_{B^{\prime}}$ where $P$ lies. On one hand, we get 
that $Y_{0} \rightarrow Y_{1} \rightarrow \cdots \rightarrow Y_{s}$ is a sectional path in $\mathcal{D}$ with infinitely many directing predecessors. On the other hand, the summand $X_{1}$ of $\operatorname{rad} P$ is not directing in $\bmod B^{\prime}$. The description of the components of $\Gamma_{B^{\prime}}$ for $B^{\prime}$ a tilted algebra (as given for example in [9]) provides the desired contradiction.

3.4. We shall now prove our main result.

Theorem. Let $A$ be a connected finite dimensional k-algebra, and let $M$ be a nonzero decomposable $A$-module such that the one-point extension $A[M]$ is quasitilted. Then every nonzero indecomposable direct summand of $M$ is directing. Moreover, $A$ is a tilted algebra.

Proof. By (1.9), it remains to show that every indecomposable direct summand of $M$ is directing. Let $M_{1}$ be an indecomposable direct summand of $M$ and assume it is not directing. Write $M=M_{1} \oplus M_{2}$. By (3.3), we get that $M_{1} \in \mathcal{L}_{A} \cap \mathcal{R}_{A}$. Moreover (1.6) implies that $M_{2}$ is a hereditary projective module in $\operatorname{add}\left(\mathcal{R}_{A}\right)$. In particular, $M_{2}$ is directing and $M \in \operatorname{add}\left(\mathcal{L}_{A} \cap \mathcal{R}_{A}\right)$. Let $Y$ be an indecomposable summand of $M_{2}$. Since $A$ is tilted (1.9), we may consider the connecting component $\mathcal{C}$. By $(3.2 . \mathrm{b}), \mathcal{C}$ is either preprojective or preinjective. Observe that $Y$ is a preprojective module. Indeed, if $Y$ is preinjective, then $\mathcal{C}$ is preinjective and $\mathcal{L}_{A} \cap \mathcal{R}_{A} \subset \mathcal{C}$, contradicting the fact that $M_{1}$ is not directing. If $Y$ lies on a nondirecting component $\mathcal{T}$, then by (1.2), $\mathcal{T} \subset \mathcal{L}_{A} \backslash \mathcal{R}_{A}$, again a contradiction. Hence $Y$ is preprojective and $Y \in \mathcal{C}$. Moreover, $\mathcal{C}$ has no injective modules because otherwise $\mathcal{L}_{A}$ would be finite. Using again (1.2), we get that $M_{1}$ lies on a regular component. By (1.3.b), there is an indecomposable module $Z$ such that $\operatorname{Hom}_{A}(Y, Z) \neq 0$ and $\operatorname{Ext}_{A}^{1}\left(M_{1}, Z\right) \neq 0$. Let $\alpha$ be a nonzero map in $\operatorname{Hom}_{A}(Y, Z)$. Then the indecomposable $B$-module $\hat{Z}=(k, Z,(0, \alpha \pi))$ where $\pi: M_{2} \longrightarrow Y$ is the canonical projection, satisfies $\operatorname{pd}_{B} \hat{Z}=2=\operatorname{id}_{B} \hat{Z}$ by (1.5). This contradiction shows our Theorem.

\section{REFERENCES}

1. M. Auslander, I. Reiten and S. Smalø, Representation theory of Artin algebras, Cambridge Studies in Advanced Mathematics 36, Cambridge Univ. Press, 1995. MR 96c:16015

2. K. Bongartz and P. Gabriel, Covering spaces in Representation Theory, Invent. Math. 65 No. 3 (1982) 331-378. MR 84i:16030

3. F. U. Coelho and D. Happel, Quasitilted algebras admit a preprojective component, Proceedings Am. Math. Soc. 125, No. 5 (1997) 1283-1291. MR 97g:16015

4. F. U. Coelho, Ma. I. R. Martins, J. A. de la Peña, Quasitilted extensions of algebras II, J. Algebra 227 (2000), 582-594.

5. F. U. Coelho and A. Skowroński, On Auslander-Reiten components for quasitilted algebras, Fundamenta Math. 149 (1996) 67-82. MR 97g:16025

6. D. Happel and C. M. Ringel, Directing projective modules, Arch. Math. 60 (1993) 237-246. MR 94b:16016

7. D. Happel and I. Reiten, Directing objects in hereditary categories, Trends in Representation Theory of Finite Dimensional Algebras, Cont. Math. 229 (1998), 169-179. CMP 99:09

8. D. Happel, I. Reiten and S. Smalø, Tilting in abelian categories and quasitilted algebras, Mem. Am. Math. Soc. 120 (1996), No. 575. MR 97j:16009

9. O. Kerner. Tilting wild algebras. J. London Math. Soc. Math. Soc. 39 (1989) $29-47$. MR 90d:16025

10. O. Kerner, Stable components of Wild tilted algebras, J. Algebra 142 (1991) 37-57. MR 92k:16021

11. H. Lenzing and A. Skowroński, Quasitilted algebras of canonical type, Coll. Math. 71 (1996) 161-181. MR 97j:16019

12. J. A. de la Peña, On the abelian Galois coverings of an algebra, J. Algebra 102 No. 1a (1986) 129-134. MR 87m:16001 
13. J. A. de la Peña and I. Reiten, Trisection of module categories, To appear.

14. I. Reiten and Ch. Riedtmann, Skew group algebras in the representation theory of artin algebras, J. Algebra 92 (1985), 224-282. MR 86k:16024

15. C. M. Ringel, Integral quadratic forms and tame algebras, Lecture Notes in Mathematics 1099 Springer (1984). MR 87f:16027

Departamento de Matemática-IMe, Universidade de São Paulo, CP 66281, São Paulo, SP, 05315-970, BRAZIL

E-mail address: fucoelho@ime.usp.br

Departamento de Matemática-IMe, Universidade de São Paulo, CP 66281, São Paulo, SP, 05315-970, BRAZIL

E-mail address: bel@ime.usp.br

Instituto de Matemáticas, UnAM, Mexico 04510 D.F., Mexico

E-mail address: jap@matem.unam.mx 